\title{
Measurement of the energy dependence of the total photon-proton cross section at HERA
}

\author{
Aharon Levy** \\ Tel Aviv University/DESY \\ E-mail: levyealzt.tau.ac.il
}

\begin{abstract}
The energy dependence of the photon-proton total cross section, $\sigma_{t o t}^{\gamma p}$, was determined from $e^{+} p$ scattering data collected with the ZEUS detector at HERA at three values of the center-of-mass energy, $W$, of the $\gamma p$ system in the range $194<W<296 \mathrm{GeV}$. This is the first determination of the $W$ dependence of $\sigma_{t o t}^{\gamma p}$ from a single experiment at high $W$. Parameterizing $\sigma_{t o t}^{\gamma p} \propto W^{2 \varepsilon}$, $\varepsilon=0.111 \pm 0.009$ (stat.) \pm 0.036 (syst.) was obtained.
\end{abstract}

The 2011 Europhysics Conference on High Energy Physics, EPS-HEP 2011,

July 21-27, 2011

Grenoble, Rhône-Alpes, France

${ }^{*}$ Speaker.

${ }^{\dagger}$ for the ZEUS Collaboration 


\section{Introduction}

The soft hadronic nature of the photon observed in $\gamma p$ collisions is well described by the vector meson dominance model, in which the photon is considered to be a superposition of vector mesons interacting with the proton. Therefore, the energy dependence above the resonance region of the total $\gamma p$ cross section, $\sigma_{t o t}^{\gamma p}$, is expected to be similar in form to that of the total hadronic cross sections, $\sigma_{\text {tot }}$, for $p p, \bar{p} p, \pi p$ and $K p$ interactions.

Donnachie and Landshoff [1] demonstrated that the energy dependences of all hadron-hadron total cross sections may be described by a simple Regge-motivated form,

$$
\sigma_{\mathrm{tot}}=A \cdot\left(W^{2}\right)^{\alpha_{\mathbb{P}}(0)-1}+B \cdot\left(W^{2}\right)^{\alpha_{R}(0)-1},
$$

where $W$ is the hadron-hadron center-of-mass energy, $A$ and $B$ are process-dependent constants, and $\alpha_{\mathbb{P}}(0)\left(\alpha_{\mathbb{R}}(0)\right)$ is process-independent and interpreted as the Pomeron (Reggeon) trajectory intercept.

At the $e p$ collider HERA, $\sigma_{t o t}^{\gamma p}$ can be extracted from $e p$ scattering at very low squared momentum transferred at the electron vertex, $Q^{2} \lesssim 10^{-3} \mathrm{GeV}^{2}$. The measurements of the total $\gamma p$ cross section at HERA for $W \simeq 200 \mathrm{GeV}[2,3,4,5,6]$ combined with measurements at low $W$ confirmed that the total photoproduction cross section has a $W$ dependence similar to that of hadron-hadron reactions. This similarity extends to virtualities $Q^{2}$ of the photon up to $\approx 1 \mathrm{GeV}^{2}$ [7].

This paper presents a determination of the $W$ dependence of $\sigma_{\text {tot }}^{\gamma p}$ from ZEUS data alone, in the range $194-296 \mathrm{GeV}$. This was made possible because in the final months of operation, the HERA collider was run with constant nominal positron energy, and switched to two additional proton energies, lower than the nominal value of $920 \mathrm{GeV}$. Many of the systematic uncertainties arising in the extraction of $\sigma_{t o t}^{\gamma p}$ are now common and do not affect the relative values of $\sigma_{t o t}^{\gamma p}$ at different $W$. As the Reggeon term is expected to be small, the function in (1.1) can be simplified to the form

$$
\sigma_{t o t}^{\gamma p}=A^{\prime} \cdot\left(\frac{W}{W_{0}}\right)^{2 \varepsilon} .
$$

This is the first extraction of the logarithmic derivative of the cross section in $W^{2}$ from a single experiment [8].

The setup of the experiment is shown in Fig. 1. At a distance of about $6 \mathrm{~m}$ from the interaction point in the ZEUS calorimeter, a spaghetti type calorimeter, the so-called six meter tagger (TAG6), was installed. The magnetic field of the HERA magnet, in which the TAG6 was located, bent the low-angle scattered positrons to the tagger, and thus the tagger was used for tagging photoproduction events. The luminosity was determined by measuring the rate of photons from the Bethe-Heitler (BH) process $\left(e^{+} p \rightarrow e^{+} \gamma p\right)$. The latter was measured by two independent systems, the photon calorimeter (PCAL) and the spectrometer (SPEC). The two components (PCAL and SPEC) enabled the measurement of the luminosity in two independent ways with $1 \%$ relative uncertainty. Overall there was a $1.8 \%$ uncertainty common to both, due to the geometrical acceptance of the exit window.

A dedicated trigger logic was designed to collect photoproduction events and keep the rates at acceptable levels. Two conditions had to be fulfilled, a low-angle scattering positron candidate detected in the TAG6 and some activity in the main detector. For further details of the analysis, including the background subtractions and photon flux calculation, see [8]. 


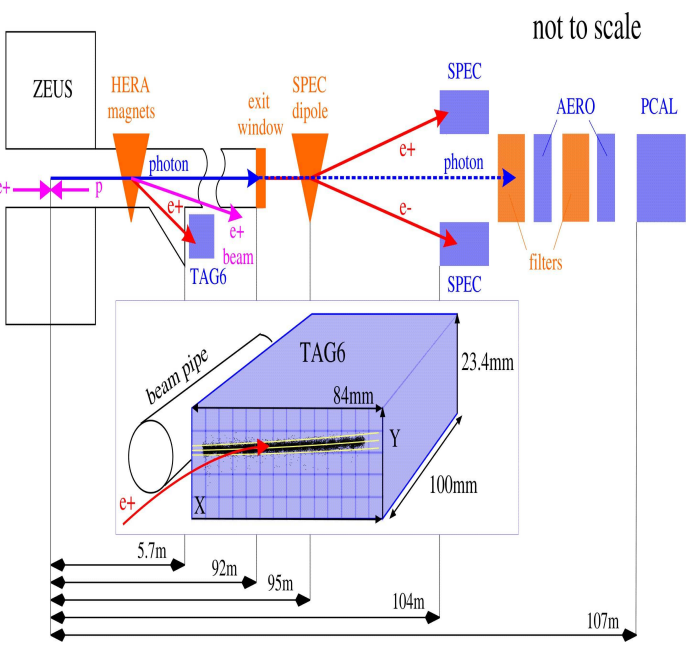

Figure 1: The layout of ZEUS and the luminosity system. To the right of the TAG6 is a side view, left of this is a top view. The inset shows the TAG6 and its cell structure in detail.
ZEUS

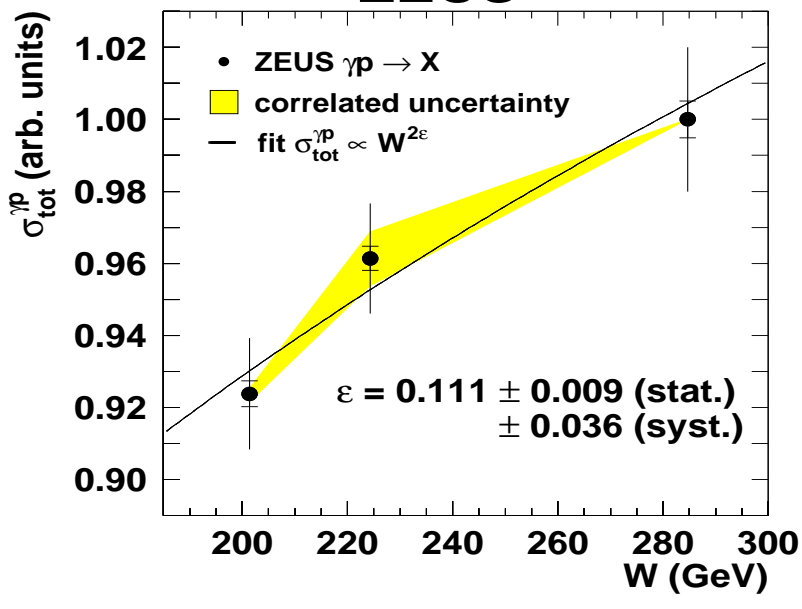

Figure 2: The $W$ dependence of the total photonproton cross section, normalized to the value for the HER. The shaded band shows the effect of the correlated systematic uncertainties. The curve shows the fit to the form $\sigma_{t o t}^{\gamma p} \propto W^{2 \varepsilon}$.

\section{Energy dependence of the total cross section}

Figure 2 shows the measured relative values of $\sigma_{t o t}^{\gamma p}$ as a function of $W$, where the cross section for HER is normalized to unity. The functional form of (1.2) was fit to the relative cross sections, with the parameter $W_{0}$ chosen to minimize correlations between the fit parameters $A^{\prime}$ and $\varepsilon$. The fit was performed using only the statistical uncertainties, and separately with all the uncorrelated systematic uncertainties added in quadrature. The correlated shifts were then applied to the data and the fit repeated; the change in $\varepsilon$ was negligible. The result for the logarithmic derivative in $W^{2}$ of the energy dependence is

$$
\varepsilon=0.111 \pm 0.009 \text { (stat.) } \pm 0.036 \text { (syst.) } .
$$

In the picture in which the photoproduction cross section is $\propto \ln ^{2}\left(W^{2}\right)$ as required by the Froissart bound, $\varepsilon \approx 0.11$ is expected, in agreement with the present measurement.

\section{References}

[1] A. Donnachie and P.V. Landshoff, Phys. Lett B 296, 227 (1992)

[2] ZEUS Coll., M. Derrick et al., Phys. Lett. B 293, 465 (1992)

[3] H1 Coll., T. Ahmed et al., Phys. Lett. B 299, 374 (1993)

[4] ZEUS Coll., M. Derrick et al., Z. Phys. C 63, 391 (1994)

[5] H1 Coll., S. Aid et al., Z. Phys C 69, 27 (1995)

[6] ZEUS Coll., S. Chekanov et al., Nucl. Phys. B 627, 3 (2002)

[7] ZEUS Coll., J. Breitweg et al., Phys. Lett. B 487, 53 (2000)

[8] ZEUS Coll., H. Abramowicz et al., Phys. Lett. B 697, 184 (2011) 